\title{
Outcomes of extended surgical resections for locally advanced thymic malignancies: a narrative review
}

\author{
Davide Tosi $^{1} \wedge$, Francesco Damarco ${ }^{1}$, Sara Franzi $^{1} \wedge$, Shehab Mohamed ${ }^{2}$, Alessandro Palleschi $^{1,2}$, \\ Paolo Mendogni ${ }^{1 \wedge}$
}

${ }^{1}$ Thoracic Surgery and Lung Transplantation Unit, IRCCS Foundation Ca' Granda Ospedale Maggiore Policlinico, Milan, Italy; ${ }^{2}$ University of Milan, Milan, Italy

Contributions: (I) Conception and design: All authors; (II) Administrative support: D Tosi; (III) Provision of study materials or patients: D Tosi, P Mendogni, A Palleschi; (IV) Collection and assembly of data: All authors; (V) Data analysis and interpretation: All authors; (VI) Manuscript writing: All authors; (VII) Final approval of manuscript: All authors.

Correspondence to: Sara Franzi, PhD. Thoracic Surgery and Lung Transplantation Unit, IRCCS Foundation Ca' Granda Ospedale Maggiore Policlinico, 20122 Milan, Italy. Email: sara.franzi@policlinico.mi.it.

Background and Objective: Thymic malignancies represent the most common anterior mediastinal neoplasms, as well as rare and challenging tumors. Surgery is the cornerstone in the treatment of thymic malignancies, although a multidisciplinary approach is mandatory, for both, locally advanced or metastatic disease. In our narrative review, we explored the recent literature to investigate clinical and radiological assessment, multimodality approach and outcomes of locally advanced thymic tumors. More than one-third of patients affected by an anterior mediastinal mass are asymptomatic at diagnosis. In case of locally advanced thymoma, symptoms are related to compression or invasion of adjacent structures, such as the superior vena cava (SVC), innominate veins and pericardium. Paraneoplastic syndromes, such as myasthenia gravis (MG), are related to release of antibodies, hormones and cytokines.

Methods: Diagnostic methods must be chosen accurately to avoid unnecessary surgical resections, to define the best strategy of care, and to plan the surgical strategy. Therefore, each case must be evaluated in a multidisciplinary context, where surgery plays an essential role.

Key Content and Findings: In this narrative review, we describe indications and surgical techniques for the treatment of locally advanced thymoma; focusing on oncological outcomes after different approaches.

Conclusions: In conclusion, aggressive surgery is always indicated, when possible, and when a complete resection can be planned, yet, the multidisciplinary approach is mandatory, in case of both locally or metastatic advanced disease.

Keywords: Thymic malignancies; mediastinal surgery; superior vena cava infiltration (SVC infiltration); multimodality treatment; myasthenia gravis (MG)

Submitted Sep 13, 2021. Accepted for publication Dec 14, 2021.

doi: $10.21037 /$ gs-21-642

View this article at: https://dx.doi.org/10.21037/gs-21-642

^ ORCID: Davide Tosi, 0000-0003-3767-0449; Sara Franzi, 0000-0003-1767-3431; Alessandro Palleschi, 0000-0003-4510-5167; Paolo Mendogni, 0000-0002-4303-6244. 


\section{Introduction}

Thymic malignancies represent the most common form of anterior mediastinal neoplasms. The most frequent histological types are thymoma, thymic carcinoma (TC), germinal tumours and primary lymphoma (1). Due to the heterogeneity of thymic malignancies, differential diagnoses have proven crucial to treat these tumours. Moreover, different treatments require a multidisciplinary evaluation (2): surgery is the cornerstone of treatment for thymic epithelial tumours (TETs), while lymphomas are usually treated with chemotherapy and radiotherapy (RT).

This narrative review investigated the clinical and the radiological assessment, the multimodality approach and outcomes of locally advanced thymic tumours.

We present the following article in accordance with the Narrative Review reporting checklist (available at https:// gs.amegroups.com/article/view/10.21037/gs-21-642/rc).

\section{Methods}

The authors carried out a non-systematic review, using PUBMED as their primary source. The search string was as follows: ("Thymoma"[Mesh:NoExp]) AND (( "locally advanced"[Mesh] OR "Thymic malignancies" [Mesh] OR "superior vena cava infiltration", OR "myasthenia gravis surgery" [Mesh])).

Articles in languages different from English and case reports were excluded. All articles retrieved were then selected by the authors based on title and abstract; the final selection was carried out after reading the full text.

\section{Clinical assessment}

More than one-third of the patients affected by an anterior mediastinal mass are asymptomatic at diagnosis (3). When evident, symptoms manifest as compression or direct invasion of surrounding structures or are associated with paraneoplastic syndromes. Timing in symptom assessment is crucial: a rapid onset of symptoms suggests the presence of an aggressive tumour, while a more indolent course indicates a slower-growing tumour. The evaluation of those signs and the symptoms associated with tumour extension, such as cough, hemoptysis, chest pain, dyspnea, voice hoarseness and superior vena cava (SVC) obstruction, has proved just as important. Vena cava obstruction, in particular, is often associated with elevated intracranial venous pressure and can result in swelling of the face, neck, upper chest, and arms, often accompanied by superficial venous distention.

Systemic symptoms result from the release of hormones, cytokines and antibodies; $30 \%$ to $60 \%$ of patients with thymoma show autoimmune manifestations. One-third of thymoma patients also suffer from myasthenia gravis (MG), the most frequent paraneoplastic-associated disease, characterised by a decreased motor response to repetitive electrical stimulation and by the presence of autoantibodies binding to anti-acetylcholine receptors (AChRs), musclespecific tyrosine kinase (MuSK), and lipoprotein receptorrelated protein 4 (LRP4). MG clinical presentation is characterised by ptosis, dysphagia, weakness, and fatigue. In contrast, thymoma was only detected in $10 \%$ to $15 \%$ of MG patients (4). In MG patients, full clinical examination, including neurological and systematic immunological check-ups, is recommended when a diagnosis of TET is suspected since these patients face an increased risk of mortality when compared to non-MG patients (5).

This complex symptomatic scenario requires an extended evaluation, which includes a complete blood count, complete metabolic panel, alpha-fetoprotein (AFP), beta-human chorionic gonadotropin (beta-hCG) and lactate dehydrogenase assessment: these tests are crucial when suspicions for non-seminomatous germ cell tumours or lymphoma arise. Moreover, a thyroid panel for thyrotoxicosis, hypercortisolism workup for Cushing syndrome and gammaglobulins count (IgG, $\operatorname{IgA}$, and $\operatorname{IgM}$ ) might prove beneficial for accurate diagnosis.

\section{Radiological assessment}

Pre-operative radiological imaging evaluation plays an essential role in the diagnosis, characterization and staging of thymic neoplasms. Moreover, a specific radiological assessment can discriminate benign masses from malignant tumours and predict the invasion of neighbouring structures by thymic tumours. These assessments are essential when determining the correct therapeutic approach (6). An initial evaluation of large anterior mediastinal masses can be performed by chest X-ray, followed by computed tomography (CT) with contrast, in order to better delineate mediastinal vascular structures that could be involved by the tumour (Figure 1).

Unfortunately, not all the lesions can be detected by traditional CT and magnetic resonance imaging (MRI); nevertheless, recent advances in imaging techniques have improved tumour characterization, the assessment 


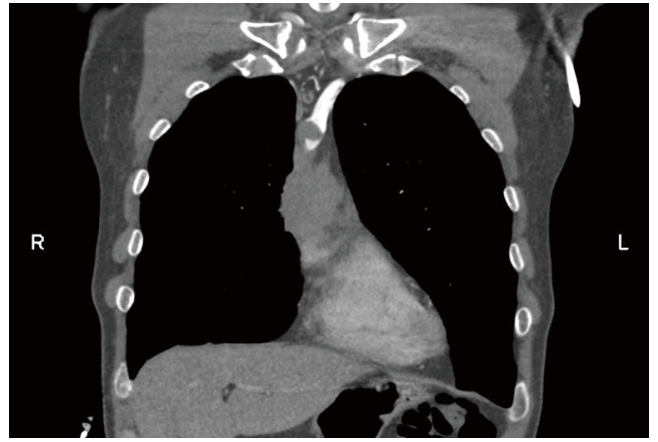

Figure 1 CT scan of Masaoka stage III thymoma invading the SVC. This image is published with the patient's consent. CT, computed tomography; SVC, superior vena cava.

of invaded adjacent structures and metastases, and the detection of lymph nodes (7).

\section{$C T$}

CT is the gold standard for the radiological assessment of thymic tumours since it ensures high sensitivity and specificity if compared to X-ray (6). CT helps to characterize lesions features, including location, size, morphology, density and enhancement characteristics; it also unveils relationships with adjacent structures and improves disease staging (8). Dual-energy CT and CT perfusion, when compared to standard CT, improve the characterization of different lesions, and give more precise information on prognosis and response to treatment (7).

\section{Fluorodeoxyglucose F18positron emission tomography/CT $\left({ }^{18}\right.$ F-FDG PET/CT)}

In recent years, ${ }^{18}$ F-FDG PET/CT has taken on major importance in the radiological evaluation of thymic malignancies, as it integrates anatomical information from CT with the metabolic data of the tumour. In particular, ${ }^{18} \mathrm{~F}$-FDG PET/CT uses 18 fluorodeoxyglucose tracer instead of a liquid contrast agent and is powerful for TCs, which are highly responsive to such tracer; in 2013, Benveniste reported TCs to have higher FDG uptake than that of thymoma, as measured by SUVmax, SUVmean, and SUVpeak. Moreover, the authors could not find any statistical association between FDG uptake and advancedstage disease for thymomas (9). For later diagnosis, 68GaDOTATE is more sensitive in distinguishing thymoma from TC, particularly in detecting metastatic lesions $(7,10)$.
Since high FDG uptake is usually associated with more aggressive tumours, PET/CT has proved to be an effective tool to distinguish thymoma from TC, but not for disease staging (7).

\section{MRI}

MRI is usually not the first choice for the radiological evaluations of locally advanced thymic tumours. Nevertheless, its higher soft-tissue contrast resolution has proven more effective than CT in differentiating cystic and thymic lesions and in assessing the infiltration of vessel, pericardium and pleura. For example, operators can rely on MRI to distinguish thymoma from thymic hyperplasia by identifying the fat inside the gland $(7,11)$.

\section{Multimodality treatment}

Thymic malignancies are rare diseases, and there is a scarcity of robust clinical trials or international accepted guidelines. Surgery remains the gold standard, although a multidisciplinary approach might improve patients' stratification and therapeutic approach, especially in stage III and IV tumours. In particular for resectable advanced tumours, radical surgery followed by adjuvant chemotherapy or RT is highly recommended. Nevertheless, in those unresectable cases, the multimodal approach might benefit the prognosis (12). The plethora of approaches described so far should be considered in a multidisciplinary context (6). Radical surgery, standard access and a miniinvasive approach (i.e., robotic thymectomy), are the mainstay of treatment for Masaoka-Koga stage I and II (6). Adjuvant RT does not seem to improve survival. On the other hand, several studies have shown the importance of multimodality treatment in Masaoka-Koga stage III and IV thymic malignancies (longer survival, lesser recurrence rate) (6). Neoadjuvant chemotherapy has the potential of tumour downstaging and prevention of systemic progression; in some cases, it has been linked to higher resectability, when compared to upfront surgery $(13,14)$. Additionally, the multimodal approach has increased the therapeutic options for stage III and IV thymoma and TCs, as well as in recurrent disease (6). Several studies have already demonstrated the high prognostic value of the multimodality approach, especially for locally advanced stage III and IV Masaoka-Koga; particularly in terms of tumour downstaging, survival rate and recurrence of disease, specifically. In 2014, Korst reported a phase II clinical 
Table 1 Stage-based therapy current treatment for locally advanced, advanced and recurrent thymic malignancies

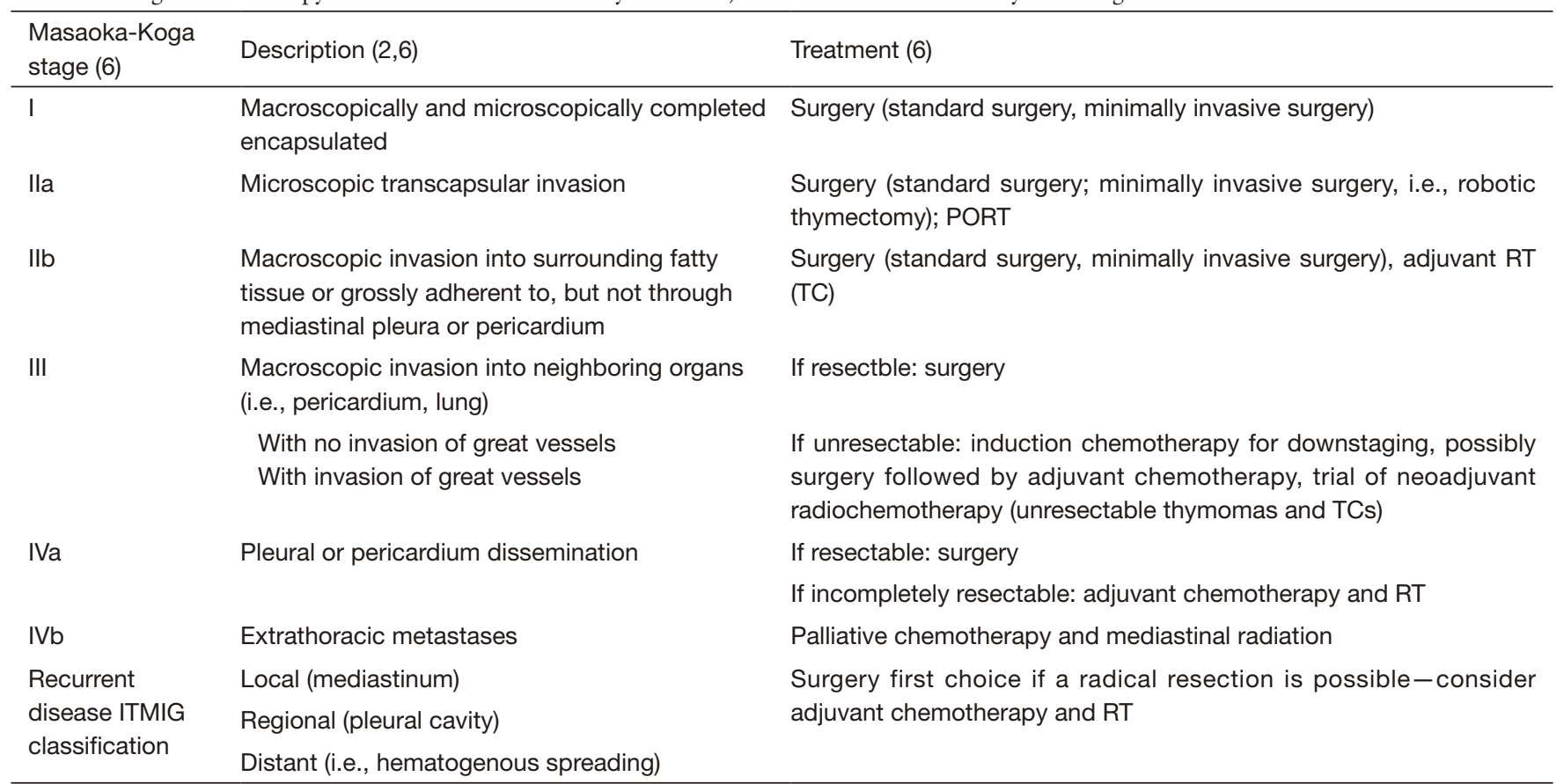

PORT, post-operative radiotherapy; RT, radiotherapy; TC, thymic carcinoma.

trial in which patients with locally advanced thymoma and TC were treated with induction chemotherapy followed by surgery: none of the patients experienced disease recurrence; after 60 months from surgery, all patients showed progression-free survival (PFS) and overall survival (OS) rates of $83 \%$ and $71 \%$, respectively (15). The optimal disease treatment strategy for Masaoka-Koga stage IV tumours is subject of even stronger debate. Although surgery remains the main prognostic factor, post-operative morbidity and mortality rate are still too elevated. In stage IVa disease, recurrence probability is high and usually entails pleural, diaphragm and pericardium involvement; thus, surgery alone might not suffice for radical removal of the tumour: in this scenario, hyperthermic intrathoracic chemotherapy (HITHOC) strategy combined with a radical pleural resection might reduce the risk of recurrence, thus ensuring effective local disease control $(6,12)$. In stage IVb tumours, usually characterised by extrathoracic metastases, surgery would not be a reasonable approach; in such rare cases, palliative chemotherapy and mediastinal radiation should be considered (6).

Multidisciplinary approach and treatment options for thymoma, TC and recurrent disease can be summarised in three forms, as shown in Table 1:

* For Masaoka-Koga stage I and II tumours, surgery (standard and minimal invasive) is the recommended strategy; for stage IIa, post-operative radiotherapy (PORT) should be considered;

* For resectable stage III tumours, surgery is the first choice. In non-resectable tumours, induction chemotherapy would be the alternative for tumour downstaging, and possibly surgery followed by chemotherapy; for totally unresectable thymoma and for TCs, chemo-RT treatment is the gold standard;

* Resectable stage IV tumours should be resected; in unresectable stage IV and stage IVb tumours, chemoRT is recommended. Palliative chemotherapy and mediastinal radiation are particularly beneficial for cases of stage $\mathrm{IVb}$ tumours;

* According to the International Thymic Malignancy Interest Group (ITMIG) classification, radical surgery is the first choice for recurrent disease; otherwise, chemotherapy and RT should be considered.

In 2020, Bruni et al. (16) published a study in which patients with stage I-IV thymoma were treated with PORT. In general, the role of PORT is controversial, although it can be beneficial for selected patients in terms of OS and disease-free survival (DFS). In detail, PORT is not 


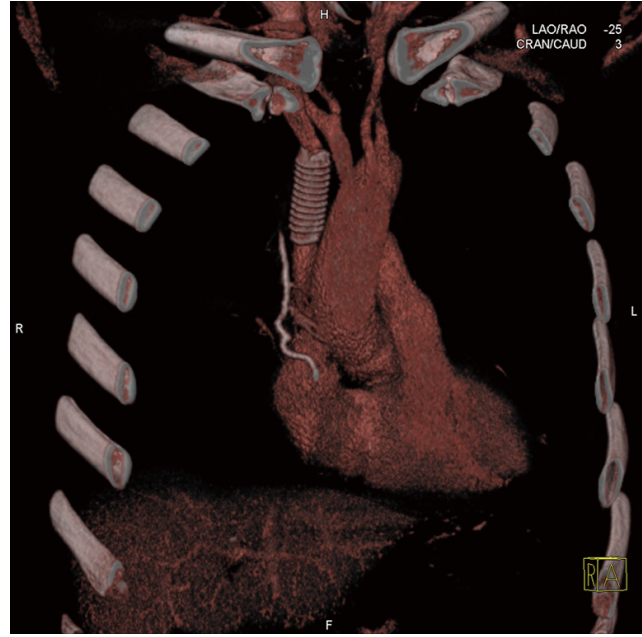

Figure 2 3D-CT coronal reconstruction of SVC prosthetic replacement. This image is published with the patient's consent. 3D-CT, three dimension computed tomography; SVC, superior vena cava.

recommended for early-stage and completely resected disease, while patients with stage III and IV seem to benefit from PORT, in terms of OS, DFS and disease-specific survival (DSS).

In the context of multidisciplinary treatment, even patients with an unresectable disease could be selected for debulking surgery followed by RT, as it was reported to be more efficient than RT alone $(17,18)$.

\section{Surgery}

The completeness of surgical resection remains the most significant factor in the prognosis of thymic malignancies, yet it is not always achievable due to the infiltration of adjacent structures (stage III) or metastatic pleural spread (stage IVa). In selected cases of locally advanced thymic malignancies, extended surgery that includes the resection of the involved organs and major vessels plays a significant role in the multimodal approach (19).

Pre-operative intravenous access should be placed below the diaphragm and in both arms to ensure functioning vascular access.

Midline sternotomy is the standard approach to explore the thorax bilaterally from mediastinum up to the phrenic nerves $(\mathrm{PN})$, as reported in the majority of the literature.

Radical resection of the thymic neoplasm, including perithymic fat tissue, thymic gland, pericardium and mediastinal pleura, is the main goal

When required, resection could be extended to the lung (wedge resection, lobectomy or pneumonectomy), PN, chest wall and great vessels (stage III).

Resection of the $\mathrm{PN}$ is particularly dreaded in myasthenic patients because of the risk of hemidiaphragmatic paralysis, which causes impairment of pulmonary function; therefore, some authors suggest using a nerve-sparing technique, which entails the skeletonization of the neural layer (12).

The effectiveness of lymph node dissections is still debated, as the incidence of lymph node metastasis in thymic malignancy has not been clearly established yet. As reported in several studies, lymphadenectomy should not affect long-term survival but seems to improve the staging as suggested by the ITMIG (20).

In case of vascular infiltration, resected vessels are closed by suturing or replaced by prostheses. SVC and the innominate vein turn are the most often involved vessels due to their features and position. The main pulmonary artery and the aorta are rarely involved.

When tumour infiltration is less than $30 \%$ of the vessel circumference, direct or pericardial patch repair is recommended. When infiltration is more than $30 \%$ or vertical extension of tumour invasion is $>2 \mathrm{~cm}$, replacement is required (Figure 2) $(21,22)$.

In case of tumour infiltration, more than $50 \%$ of the authors suggest performing prosthetic reconstruction $(23,24)$.

Various materials have been used for revascularization. Synthetic prostheses are still the most popular material for vascular replacement: in particular, polytetrafluoroethylene (PTFE) grafts are easily adjustable in size and length and can be quickly re-epithelised, while the prosthetic ring prevents collapse by maintaining good patency, despite the need for long-term anticoagulation therapy. Biological autologous grafts (for e.g., bovine pericardium/ jugular vein, porcine pericardium, saphenous vein) can be used for venous replacement since they provide better biocompatibility with no need for anticoagulation therapy but require a longer time for preparation than synthetic prostheses do.

In case of venous infiltration, surgical manoeuvres are usually performed by the cross-clamping technique. Operators should always bear in mind that sudden clamping could decrease preload and cardiac output as well as lead to systemic hypotension. It also could increase venous pressure, which can result in brain oedema and cerebral damages. A complete vessel clamping is usually tolerated 
for up to 45-60 minutes with appropriate pharmacological support for neuroprotection such as steroids, massive fluid administration and vasoconstrictive agents.

Otherwise, a temporary shunt between brachiocephalic trunks and the right atrium allows the stabilization of hemodynamic parameters, as shown by Matsumoto et al. (25).

In case of vascular replacement, intravenous anticoagulant therapy is routinely administrated during surgery. Low-molecular-weight heparin is administered after surgery and then shifted to oral anticoagulation at discharge.

The identification of the optimal strategy remains controversial, and the replacement strategy differs depending on neoplasm extension.

In case of SVC trunk infiltration, vascular reconstruction is performed between the two venous stumps with an endto-end anastomosis after clamping and vascular resection.

In case of infiltration at the confluence with brachiocephalic trunks, different approaches are hereby proposed:

* Vascular reconstruction between the left brachiocephalic vein and the inferior SVC stump or the right atrium with the closure of the right brachiocephalic vein;

* Vascular reconstruction between the right brachiocephalic vein and the inferior SVC stump or the right atrium with the closure of the left brachiocephalic vein;

* Using a double replacement between the left brachiocephalic vein and the inferior SVC stump or the right atrium and the right brachiocephalic vein and the inferior SVC stump or the right atrium.

* Using a Y-shaped prosthesis between the two brachiocephalic trunks and the inferior SVC stump or the right atrium (26).

The review of the existing literature provides operators with a range of recommendations and arrangements to reduce post-operative complications:

- SVC is a high flow and low-pressure system: therefore, it is important to mimic physiological conditions after vascular replacement in order to maintain prosthetic patency. Sekine et al. (23) suggest using smaller sized grafts, whereas many authors have abandoned Y-shaped reconstructions or single left brachiocephalic vein grafts due to the higher tendency to occlude, as reported by Shintani et al. (27);

* Soaking the ringed PTFE prosthesis in heparin-salt solution minimises thrombotic risk (28);
* If total SVC clamping is needed during operation, SVC should be clamped above the confluence of the azygos vein to preserve collateral circulation. If needed, a clamp below the azygos vein or revascularization are supposed to last longer than 45-60 minutes; temporary SVC shunt or cardiopulmonary bypass $(\mathrm{CPB})$ should be considered to avoid brain damages.

If the right side of the heart, specifically the aorta or the supra-aortic trunks, are infiltrated, CPB support is mandatory. $\mathrm{CPB}$ can be established with aortic or femoral artery cannulation, and through right atrium or femoral vein drainage. Heparin $(350 \mathrm{IE} / \mathrm{kg})$ and mild to moderate hypothermia are the most common settings. Hypothermic CPB and cardioplegic cardiac arrest have also been reported (29).

Overall, only a few reports describe ascending aorta or aortic arch replacement; such scarcity is due to the extreme surgical challenges posed by the procedure (29-31).

In case of pleural metastases (stage IVa) surgical options range from regional or complete parietal pleurectomy to extrapleural pleuropneumonectomy (EPP); yet, the benefits of the latter are still under debate (32).

The most frequent post-operative complications are bleeding, respiratory and cardiac arrhythmic events (11.1\% in Maurizi, 23\% in Spaggiari and 25\% in Sekine studies, respectively) $(21,23,24)$.

The reported post-operative mortality rate varies from $0 \%$ to $7.7 \%$; these numbers probably reflect the extension of the surgery, the need for CPB and careful patients' selection.

Graft patency is another significant achievement for patients' health: conduit occlusion is one of the most common problems and widely differs among literature. According to the recommendations listed above, longlasting anticoagulant therapy is useful to prevent this type of complication.

Categories of cancer, their histotypes and the completeness of surgical resection are the main long-term prognostic factors: they affect local recurrence, distant metastases and, consequently, survival rates.

Disease recurrence can involve all thoracic structures or distant sites, and pleural space is the most common target (about 75\%). Nevertheless, thymoma, thymic carcinoid and TC show different patterns in recurrence locations (regional or distant), in cancer-free interval and biological behaviour.

Several studies describe their experience on surgery for recurrence, but it is hard to identify the optimal approach. Completeness of resection is the most important long-term survival prognostic factor in repeated surgery for recurrent 
malignancies as well: therefore, surgical treatments should be restricted to selected patients with limited locoregional recurrence within the context of multimodal therapies (33).

\section{Oncological outcomes}

Complete surgical resection is considered the most important prognostic factor. However, complete tumour resection is not often achievable for stage III thymomas. Thus, surgeons recommend resection only in association with chemo-RT prior to or after surgery. Here, we reviewed current literature to collect relevant data on oncologic outcomes in patients with stage III thymic tumours (Table 2).

Fan et al. (17) followed a cohort of 82 patients with Masaoka-Koga stage III thymoma on average 41 months (range, 5-166 months), subjected to debulking surgery or biopsy and treated or not with definitive RT (dRT) (dRT group and non-dRT group, respectively). They found that dRT significantly improved 5 - and 10-year OS rate $(65.7 \%$ and $55.8 \%$, respectively) $v s$. non-dRT group $(26.8 \%$ and $13.4 \%$, respectively, $\mathrm{P}=0.008$ ). Similarly, 5- and 10-year PFS was higher in dRT group ( $46.1 \%$ and $34.6 \%$, respectively) compared to non-dRT $(17.0 \%$ and $0 \%$, respectively) $(\mathrm{P}=0.003)$ (Table 2).

In the same way, Funaki et al. (38) reported that the combination of induction therapy and surgical resection could improve the outcome of patients with advanced locally invasive thymoma.

The beneficial role of PORT in patients with stage III thymoma has been widely described (16). Lim et al. (34) reported in 2016 potential OS benefit in patients who received PORT in stage III and IV disease (HR $=0.63 ; 95 \%$ CI: 0.40-0.99; $\mathrm{P}=0.04$ ) (Table 2). Similarly, a meta-analysis from Zhou et al. (35) in 2016 found an OS improvement by PORT in completely resected stage III thymoma patients (HR $=0.73$ ) but no statistical improvements for DFS. Alothaimeen and colleagues reviewed 56 patients with thymoma and TC and reported 5- and 10-year DFS rates as $69.9 \%$ and $43.8 \%$, respectively. Accordingly, 5- and 10-year OS rates were $88.6 \%$ and $74.3 \%$, respectively. The Kaplan Meyer analysis of OS according to histologic classification showed a median OS of 61 months for thymoma and 84 months for TC. More specifically, the 5- and 10-year OS rates for thymoma were $90 \%$ and $84 \%$, while for TC were $75 \%$ and $25 \%$, respectively (39). In their original research, Tian and colleagues evaluated oncological prognosis in patients with TETs, associated or not with MG. Patients with MG were subjected to extended thymectomy with the removal of the entire thymus and mediastinal fat tissue bilaterally. Patients without MG received thymectomy associated with the removal of adjacent lymph nodes. Over a median of 42 months follow-up, the 5-year OS rate was $88.6 \%$, and there was no difference in the prognosis between MG and no-MG patients (Table 2) (36).

Great-vessels invasion was reported as a poor prognostic factor (40). In this regard, several papers were published on the surgery approaches for advanced thymic tumours with great vessel invasion.

In 2019 Maurizi et al. (21) reported on the outcomes of patients subjected to radical resection with vessel reconstruction for advanced thymic tumours infiltrating SVC. Over 58 months follow-up, 3- and 5-year survival rates were $80 \%$ and $58.1 \%$, while 3 - and 5 -year DFS rates were $90.5 \%$ and $67 \%$, respectively. In particular, 3 - and 5-year survival rates of stage III thymoma were $100 \%$ and $93.8 \%$, compared to TC, $50 \%$ and $0 \%$, respectively (Table 2).

More recently, Yu et al. (41) reported on long-term outcomes in patients subjected to tumour resection with SVC replacement for advanced thymoma, receiving neoadjuvant chemotherapy or RT. Kaplan Meier analysis showed better recurrence-free survival curves for patients treated with the combination cardiac resynchronization therapy $(\mathrm{CRT})+$ surgery, with respect to the only surgery group $(\mathrm{P}=0.031)$. Nevertheless, there were no statistical differences between the two groups for OS $(\mathrm{P}=0.069)$.

Similarly, research from Liu and colleagues showed that extended thymectomy associated with vessel resection and reconstruction improves clinical outcomes in patients with stage III TC when compared to non-surgery patients. Over a median follow-up of 44 months, the authors found a survival rate in the operative group of $66.67 \%$ with respect to the non-operative group, $35.71 \%$. The median OS was of 48 and 26 months in the two groups, respectively $(\mathrm{P}=0.013)$. The overall disease metastasis-free survival were 47 and 18 months in the operative and non-operative groups, respectively ( $\mathrm{P}=0.019$ ) (Table 2) (42). In 2019, Aprile et al. (37) published a retrospective study describing a nervesparing surgery to treat patients with invasive advanced thymoma (stage III and IV). Over a median follow-up of 101 months (range, 15-323 months), OS was 273.4 months for stage III thymoma (95\% CI: 229-318) with respect to 159.2 months of stage IVa thymoma (95\% CI: 106-211). In the total cohort, 5- and 10-year survival rates were $88 \%$ and $78 \%$, respectively. DFS was statistically higher for stage III than for stage IVa thymoma (287.6 vs. 28 months, respectively; $\mathrm{P}<0.001)$ (Table 2). 


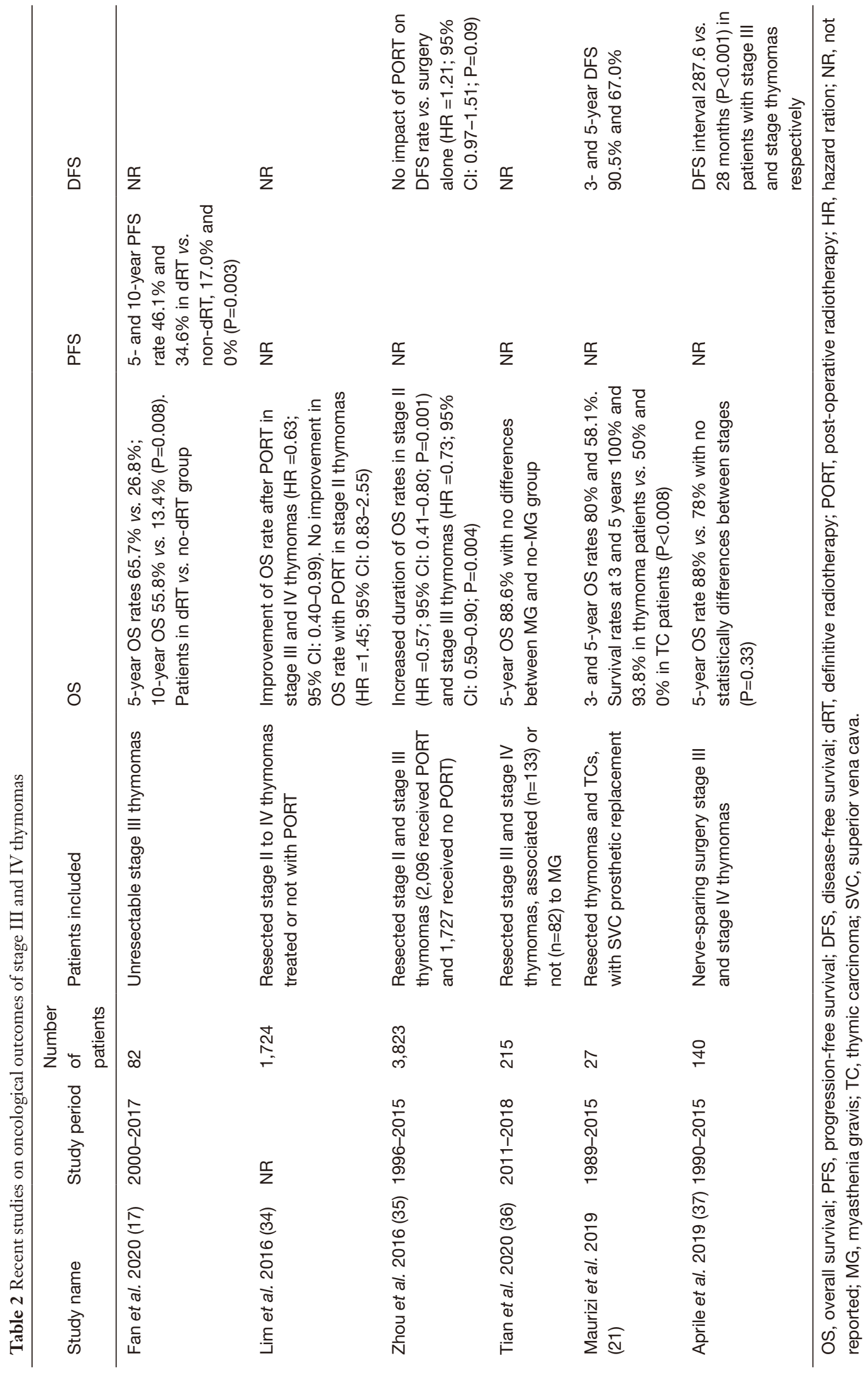




\section{Discussion}

The surgery of advanced stage thymoma is one of the main challenges for thoracic surgeons and thoracic oncologists. To date, the main issue remains the lack of precise guidelines or recommendations regarding the multimodal approach and the pre-operative assessment of the malignancy. Due to the high peri-operative risks and the uncertain oncological outcomes, most of the patients with locally advanced TETs do not undergo surgery, especially if metastases have invaded the heart and/or the great mediastinal vessels. Reported results are not straightforward and prevent the authors from drawing any firm conclusion and from clarifying when to opt for either surgical or nonsurgical treatments.

Despite the multicentric nature of the studies considered, the total number of patients in the international cohort is still too small to give solid foundations for future randomised studies.

Each centre has different treatment protocols in place, especially for the pre-operative neo-adjuvant therapies. The option of multimodal treatment must always be taken into consideration in view of the relatively high chemosensitivity and radiosensitivity of these neoplasms. In particular, neoadjuvant therapies should be considered to reduce the mass and to improve the resectability of the tumour, in case of high invasiveness. Nevertheless, it has been acknowledged that surgery is the gold standard in the treatment of these rare and challenging neoplasms, even in those cases where surgery cannot guarantee complete tumour resection (R1 or R2 resections). Debulking surgery followed by RT has been reported to be more efficient than RT alone, yet there is no indisputable evidence on the matter in current literature $(17,18)$. Oncologic results are encouraging: a complete resection must be pursued for better long-term results about recurrence and survival. Despite a radical resection, the possibility of local and distance recurrence is not insignificant. Furthermore, an incomplete resection is associated with a high recurrence rate in advanced thymomas. As reported by Marulli et al. (43), the most important prognostic factors in resectable stage III tumours are the completeness of resection, the type and grade of surrounding organs infiltration and recurrence.

In addition, it is necessary to make a distinction between thymoma and TC. These two different subtypes of TETs show extremely different aggressiveness and invasive behaviour, being thymomas more "indolent" and rarely infiltrating or spreading metastasis; on the contrary, TC is usually diagnosed when already advanced and often with distant metastasis. Good short and long-term results confirm that, after a careful multidisciplinary discussion, surgery should be the first choice even in selected advanced TC. In some reported series, thymoma and TC have not been considered separately, leading to contradictory and inhomogeneous results. The literature shows that when the great vessels are involved and reconstructions or resections are required, surgery is not always an option for locally advanced cases.

The lack of experience and skills of the surgical and anaesthesiology staff in low-volume centres could lead to reducing the surgical indications, especially if extracorporeal life support is required.

The CPB support for this type of surgery is not routinely used in the majority of thoracic surgery centres all around the world; therefore, the presence of a cardiac surgery service is mandatory for the complexity of patients as well as the skills in the use of CPB.

Another critical issue is the surgical therapy in cases of pleural dissemination (stage IV) or for pleural relapses after surgery: Moser et al. (44) suggest that surgery should be considered as a part of the multimodal treatment.

The main limitation of this paper is due to nonsystematic review of the literature; nevertheless, we can conclude that aggressive surgery is always indicated when a complete resection proves feasible. The multidisciplinary approach is mandatory in every case of both locally or metastatic advanced TETs.

\section{Acknowledgments}

Funding: The cover publication costs were supported by a fund dedicated to research derived from the Italian Ministry of Health donated to the Fondazione IRCCS Ca' Granda Ospedale Maggiore Policlinico.

\section{Footnote}

Reporting Checklist: The authors have completed the Narrative Review reporting checklist. Available at https:// gs.amegroups.com/article/view/10.21037/gs-21-642/rc

Peer Review File: Available at https://gs.amegroups.com/ article/view/10.21037/gs-21-642/prf

Conflicts of Interest: All the authors have completed the ICMJE uniform disclosure form (available at https:// 
gs.amegroups.com/article/view/10.21037/gs-21-642/coif). The authors have no conflicts of interest to declare.

Ethical Statement: The authors are accountable for all aspects of the work in ensuring that questions related to the accuracy or integrity of any part of the work are appropriately investigated and resolved.

Open Access Statement: This is an Open Access article distributed in accordance with the Creative Commons Attribution-NonCommercial-NoDerivs 4.0 International License (CC BY-NC-ND 4.0), which permits the noncommercial replication and distribution of the article with the strict proviso that no changes or edits are made and the original work is properly cited (including links to both the formal publication through the relevant DOI and the license). See: https://creativecommons.org/licenses/by-nc-nd/4.0/.

\section{References}

1. Marx A, Ströbel P, Badve SS, et al. ITMIG consensus statement on the use of the WHO histological classification of thymoma and thymic carcinoma: refined definitions, histological criteria, and reporting. J Thorac Oncol 2014;9:596-611.

2. NCCN. NCCN Clinical Practice Guidelines in Oncology (NCCN Guidelines®) Thymomas and Thymic Carcinomas Version 1.2021. Available online: https://www. nccn.org/

3. Aroor AR, Prakasha S R, Seshadri S, et al. A study of clinical characteristicsof mediastinal mass. J Clin Diagn Res 2014;8:77-80.

4. Álvarez-Velasco R, Gutiérrez-Gutiérrez G, Trujillo JC, et al. Clinical characteristics and outcomes of thymoma-associated myasthenia gravis. Eur J Neurol 2021;28:2083-91.

5. Hansen JS, Danielsen DH, Somnier FE, et al. Mortality in myasthenia gravis: A nationwide population-based followup study in Denmark. Muscle Nerve 2016;53:73-7.

6. Ried M, Marx A, Götz A, et al. State of the art: diagnostic tools and innovative therapies for treatment of advanced thymoma and thymic carcinoma. Eur J Cardiothorac Surg 2016;49:1545-52.

7. Gentili F, Monteleone I, Mazzei FG, et al. Advancement in Diagnostic Imaging of Thymic Tumors. Cancers (Basel) 2021;13:3599.

8. Carter BW, Benveniste MF, Marom EM. Diagnostic approach to the anterior/prevascular mediastinum for radiologists. Mediastinum 2019;3:18.

9. Benveniste MF, Moran CA, Mawlawi O, et al. FDG PETCT aids in the preoperative assessment of patients with newly diagnosed thymic epithelial malignancies. J Thorac Oncol 2013;8:502-10.

10. Hephzibah J, Shanthly N, Oommen R. Diagnostic Utility of PET CT in Thymic Tumours with Emphasis on 68GaDOTATATE PET CT in Thymic Neuroendocrine Tumour - Experience at a Tertiary Level Hospital in India. J Clin Diagn Res 2014;8:QC01-3.

11. Priola AM, Priola SM, Ciccone G, et al. Differentiation of rebound and lymphoid thymic hyperplasia from anterior mediastinal tumors with dual-echo chemical-shift MR imaging in adulthood: reliability of the chemical-shift ratio and signal intensity index. Radiology 2015;274:238-49.

12. Aprile V, Bacchin D, Korasidis S, et al. Hypertermic Intrathoracic Chemotherapy (HITHOC) for thymoma: a narrative review on indications and results. Ann Transl Med 2021;9:957.

13. Park S, Park IK, Kim Y'T, et al. Comparison of Neoadjuvant Chemotherapy Followed by Surgery to Upfront Surgery for Thymic Malignancy. Ann Thorac Surg 2019;107:355-62.

14. Cardillo G, Carleo F, Giunti R, et al. Predictors of survival in patients with locally advanced thymoma and thymic carcinoma (Masaoka stages III and IVa). Eur J Cardiothorac Surg 2010;37:819-23.

15. Korst RJ, Bezjak A, Blackmon S, et al. Neoadjuvant chemoradiotherapy for locally advanced thymic tumors: a phase II, multi-institutional clinical trial. J Thorac Cardiovasc Surg 2014;147:36-44,46.e1.

16. Bruni A, Stefani A, Perna M, et al. The role of postoperative radiotherapy for thymomas: a multicentric retrospective evaluation from three Italian centers and review of the literature. J Thorac Dis 2020;12:7518-30.

17. Fan C, Ge H, Zhang S, et al. Impact of Definitive Radiotherapy and Surgical Debulking on Treatment Outcome and Prognosis for Locally Advanced MasaokaKoga stage III Thymoma. Sci Rep 2020;10:1735.

18. Zhai Y, Hui Z, Gao Y, et al. Debulking Surgery Plus Radiation: Treatment Choice for Unresectable Stage III Thymic Carcinoma. Thorac Cardiovasc Surg 2020;68:440-5.

19. Venuta F, Anile M, Diso D, et al. Thymoma and thymic carcinoma. Eur J Cardiothorac Surg 2010;37:13-25.

20. Hwang Y, Kang CH, Park S, et al. Impact of Lymph Node Dissection on Thymic Malignancies: Multi-Institutional Propensity Score Matched Analysis. J Thorac Oncol 2018;13:1949-57. 
21. Maurizi G, Poggi C, D'Andrilli A, et al. Superior Vena Cava Replacement for Thymic Malignancies. Ann Thorac Surg 2019;107:386-92.

22. Kumar A, Pulle MV, Asaf BB, et al. Superior Vena Cava Resection in Locally Advanced Thymoma-Surgical and Survival Outcomes. Indian J Surg Oncol 2020;11:711-9.

23. Sekine Y, Suzuki H, Saitoh Y, et al. Prosthetic reconstruction of the superior vena cava for malignant disease: surgical techniques and outcomes. Ann Thorac Surg 2010;90:223-8.

24. Spaggiari L, Leo F, Veronesi G, et al. Superior vena cava resection for lung and mediastinal malignancies: a single-center experience with 70 cases. Ann Thorac Surg 2007;83:223-9; discussion 229-30.

25. Matsumoto K, Yamasaki N, Tsuchiya T, et al. Temporary bypass for superior vena cava reconstruction with Anthron bypass tubeTM. J Thorac Dis 2017;9:E614-8.

26. Chen KN, Xu SF, Gu ZD, et al. Surgical treatment of complex malignant anterior mediastinal tumors invading the superior vena cava. World J Surg 2006;30:162-70.

27. Shintani Y, Ohta M, Minami M, et al. Long-term graft patency after replacement of the brachiocephalic veins combined with resection of mediastinal tumors. J Thorac Cardiovasc Surg 2005;129:809-12.

28. Sun Y, Gu C, Shi J, et al. Reconstruction of mediastinal vessels for invasive thymoma: a retrospective analysis of 25 cases. J Thorac Dis 2017;9:725-33.

29. Ried M, Neu R, Schalke B, et al. Radical surgical resection of advanced thymoma and thymic carcinoma infiltrating the heart or great vessels with cardiopulmonary bypass support. J Cardiothorac Surg 2015;10:137.

30. Yamato H, Funaki S, Shimamura K, et al. Salvage surgery for stage IVa thymic carcinoma combined with aortic arch resection - case report. J Cardiothorac Surg 2020;15:305.

31. Kuno H, Funaki S, Kimura K, et al. Complete resection of local advanced thymic carcinoma with total aortic arch replacement after chemotherapy: a case report. Surg Case Rep 2019;5:198.

32. Markowiak T, Ansari MKA, Neu R, et al. Evaluation of Surgical Therapy in Advanced Thymic Tumors. Cancers (Basel) 2021;13:4516.

33. Hamaji M, Allen MS, Cassivi SD, et al. The role of surgical management in recurrent thymic tumors. Ann Thorac Surg 2012;94:247-54; discussion 254.

34. Lim YJ, Kim E, Kim HJ, et al. Survival Impact of Adjuvant Radiation Therapy in Masaoka Stage II to IV Thymomas: A Systematic Review and Meta-analysis. Int J Radiat Oncol
Biol Phys 2016;94:1129-36.

35. Zhou D, Deng XF, Liu QX, et al. The Effectiveness of Postoperative Radiotherapy in Patients With Completely Resected Thymoma: A Meta-Analysis. Ann Thorac Surg 2016;101:305-10.

36. Tian W, Sun Y, Wu Q, et al. Surgical outcomes of 215 patients with thymic epithelial tumors: A single-center experience. Thorac Cancer 2020;11:1840-7.

37. Aprile V, Bertoglio P, Korasidis S, et al. Nerve-Sparing Surgery in Advanced Stage Thymomas. Ann Thorac Surg 2019;107:878-84.

38. Funaki S, Shintani Y, Fukui E, et al. Surgical treatment strategies for invasive thymoma. J Thorac Dis 2020;12:7619-25.

39. Alothaimeen HS, Memon MA. Treatment Outcome and Prognostic Factors of Malignant Thymoma - A Single Institution Experience. Asian Pac J Cancer Prev 2020;21:653-61.

40. Yamada Y, Yoshino I, Nakajima J, et al. Surgical Outcomes of Patients With Stage III Thymoma in the Japanese Nationwide Database. Ann Thorac Surg 2015;100:961-7.

41. Yu Z, Yu L, Yu T, et al. Surgical feasibility and longterm outcome of superior vena cava replacement for advanced thymoma in patients undergoing preoperative chemotherapy or chemoradiotherapy. Thorac Cancer 2021;12:1074-83.

42. Liu L, Zhang J, Wang G, et al. Extended thymectomy with blood vessel resection and reconstruction improves therapeutic outcome for clinical stage III thymic carcinoma patients: a real-world research. J Cardiothorac Surg 2020;15:267.

43. Marulli G, Lucchi M, Margaritora S, et al. Surgical treatment of stage III thymic tumors: a multi-institutional review from four Italian centers. Eur J Cardiothorac Surg 2011;39:e1-7.

44. Moser B, Fadel E, Fabre D, et al. Surgical therapy of thymic tumours with pleural involvement: an ESTS Thymic Working Group Project. Eur J Cardiothorac Surg 2017;52:346-55.

Cite this article as: Tosi D, Damarco F, Franzi S, Mohamed S, Palleschi A, Mendogni P. Outcomes of extended surgical resections for locally advanced thymic malignancies: a narrative review. Gland Surg 2022;11(3):611-621. doi: 10.21037/gs-21642 